\title{
Actin Is a Target of T-Cell Reactivity in Patients with Advanced Carotid Atherosclerotic Plaques
}

\author{
Elisabetta Profumo, ${ }^{1}$ Brigitta Buttari, ${ }^{1}$ Linda Petrone, ${ }^{1}$ Giada Lacroce, ${ }^{2}$ \\ Maria Chiara Tesori, ${ }^{2}$ Raffaele Capoano, ${ }^{2}$ Bruno Salvati, ${ }^{2}$ and Rachele Riganò ${ }^{1}$ \\ ${ }^{1}$ Department of Infectious, Parasitic and Immune-Mediated Diseases, Istituto Superiore di Sanità, 299 Viale Regina Elena, \\ 00161 Rome, Italy \\ ${ }^{2}$ Department of Surgical Sciences, Sapienza University of Rome, 155 Viale del Policlinico, 00161 Rome, Italy
}

Correspondence should be addressed to Rachele Riganò; rachele.rigano@iss.it

Received 30 July 2013; Accepted 7 October 2013

Academic Editor: Fatih Arslan

Copyright (C) 2013 Elisabetta Profumo et al. This is an open access article distributed under the Creative Commons Attribution License, which permits unrestricted use, distribution, and reproduction in any medium, provided the original work is properly cited.

\begin{abstract}
Atherosclerosis is a chronic inflammatory disease of the arterial wall associated with autoimmune reactions. In a previous study, we observed the presence of actin-specific antibodies in sera from patients with carotid atherosclerosis. To extend our previous results we evaluated the possible role of actin as antigenic target of cell-mediated immune reactions in carotid atherosclerosis. Peripheral blood mononuclear cells (PBMC) from 17 patients and 16 healthy subjects were tested by cell proliferation assay and by ELISA for cytokine production. Actin induced a proliferative response in 47\% of patients' PBMC samples, with SI ranging from 2.6 to 21.1, and in none of the healthy subjects' samples (patients versus healthy subjects, $P=0.02$ ). The presence of diabetes in patients was significantly associated with proliferative response to actin $(P=0.04)$. IFN- $\gamma$ and TNF- $\alpha$ concentrations were higher in PBMC from patients than in those from healthy subjects and in PBMC proliferating to actin than in nonproliferating ones. Our data demonstrate for the first time a role of actin as a target autoantigen of cellular immune reactions in patients with carotid atherosclerosis. The preferential proinflammatory Thl activation suggests that actin could contribute to endothelial dysfunction, tissue damage, and systemic inflammation in carotid atherosclerosis.
\end{abstract}

\section{Introduction}

Atherosclerosis is a chronic inflammatory disease of the arterial wall in which immune responses play a crucial role. Atherosclerotic plaques are characterized by the presence of an inflammatory cell infiltrate mainly composed of macrophages and $\mathrm{T}$ lymphocytes that modulate the atherosclerotic process by secreting inflammatory mediators. Infiltrating $\mathrm{T}$ lymphocytes are activated $\mathrm{T}$ cells expressing CD25 on their surface [1] and predominantly expressing a Th1 phenotype in advanced lesions $[2,3]$. In this context, identifying the antigens responsible for $\mathrm{T}$ lymphocyte activation in atherosclerosis may be relevant. Accelerated atherosclerosis has been reported in patients with various autoimmune diseases [4-6], suggesting an involvement of autoimmune mechanisms in atherogenesis [7]. Although infectious agents have been associated with the activation of immune mechanisms, several lines of evidence suggest that the main antigenic targets in atherosclerosis are modified endogenous structures [8]. Different self-antigens or modified self-molecules have been identified as target of humoral and cellular immune responses in patients with atherosclerotic disease thus behaving as dangerous signals able to activate proinflammatory responses. Oxidative stress, increasingly reported in these patients [9], is the major event causing structural modification of proteins [10].

Oxidized low density lipoproteins (LDL) are the best characterized autoantigen. In particular, it has been demonstrated that about $10 \%$ of $\mathrm{T}$ lymphocytes infiltrating human atherosclerotic plaques are specific for oxidized LDL [11]. In addition to LDL, other self-molecules modified by oxidative stress become target of autoimmune reaction in atherosclerosis [12-14]. Another two categories of autoantigens that have been implicated in atherosclerosis are the stress-induced 


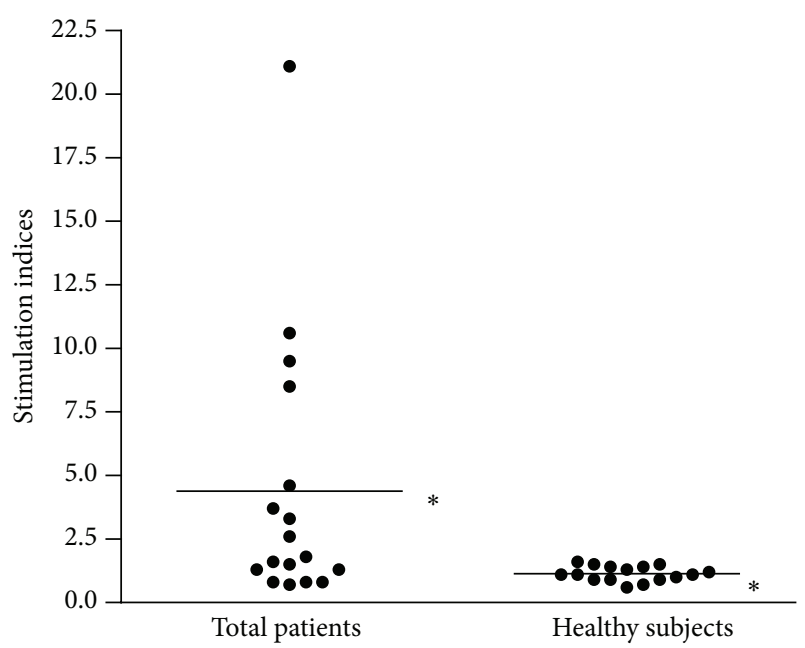

Figure 1: Proliferative response of peripheral blood mononuclear cell samples obtained from the 17 patients with carotid atherosclerosis and from the 16 healthy subjects. ${ }^{*} P=0.02$.

2.5. Statistical Analysis. Results are expressed as arithmetic means or medians and interquartile ranges. Mann-Whitney $U$ and Wilcoxon nonparametric tests were used to investigate the significance of unpaired and paired data. All the covariates were examined in univariate analyses as predictors for actin-specific cellular response. Fisher's exact test and Mann-Whitney $U$ test were used to evaluate the differences in discrete and continuous clinical characteristics between patients' groups. A $P$ value less than 0.05 was considered statistically significant.

\section{Results}

3.1. Proliferative Response of Circulating T Lymphocytes to Actin. In our selected healthy subject population, we determined a mean SI value of 1.13 and a SD of 0.29 and we calculated the value of 2.0 as the cutoff level for positivity. Actin induced a proliferative response in 8 of 17 (47\%) patients' PBMC samples, with SI ranging from 2.6 to 21.1 (Figure 1). PBMC samples from healthy subjects did not proliferate in response to actin. The difference between the SI mean values in patients and healthy subjects was statistically significant (4.4 versus $1.1, P=0.02$ by Mann-Whitney $U$ test). Univariate analysis showed that the presence of diabetes in patients was significantly associated with proliferative response to actin $(P=0.04$, Figure 2$)$.

3.2. Cytokine Production. PBMC samples from patients produced higher concentrations of IFN- $\gamma$ and TNF- $\alpha$ than PBMC from healthy subjects (Figure 3). In patients, IFN- $\gamma$ and TNF- $\alpha$ concentrations were higher in PBMC samples that proliferated in response to actin than in nonproliferating ones (Figure 3). No significant differences were observed for IL-4 and IL-10 production (Figure 3 ). We found the presence of a positive correlation between IFN- $\gamma$ concentrations and SI $\left(P<10^{-4} ; r=0.71\right)$ (Figure 4).

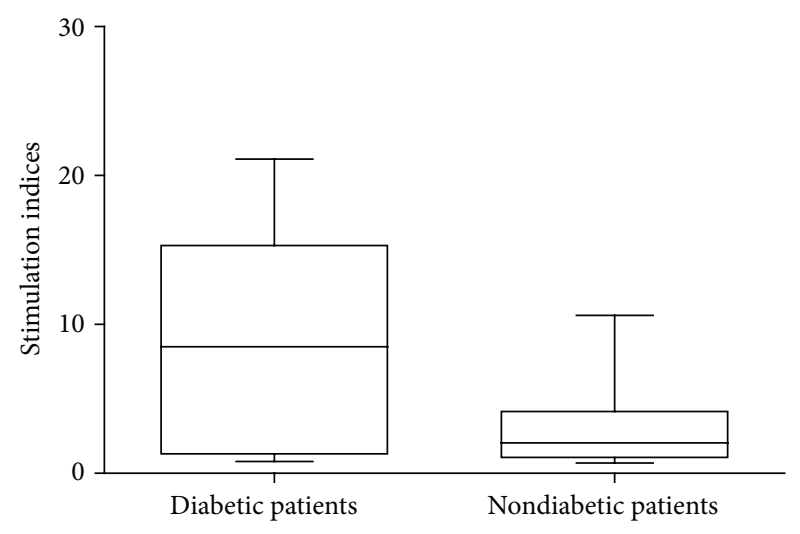

FIGURE 2: Proliferative response of peripheral blood mononuclear cell samples obtained from the 17 patients with carotid atherosclerosis divided according to the presence/absence of type 2 diabetes. ${ }^{*} P=0.04$.

\section{Discussion}

In this study, we demonstrated for the first time a role of actin as a target autoantigen of cellular immune reactions in patients with carotid atherosclerosis. As observed for other candidate autoantigens, actin induced a proinflammatory Thl activation, characterized by high IFN- $\gamma$ and TNF- $\alpha$ expression. Th1 response, not counteracted by an increase of anti-inflammatory IL- 4 and IL-10 production, may contribute to tissue damage, endothelial dysfunction, and systemic inflammation [12]. Further characterization of these will establish whether they are a regulatory population able to counteract. Our results support previous findings indicating that inflammatory autoimmune reactions are not exclusively localized within atherosclerotic lesions but can also contribute to systemic inflammation in patients with atherosclerosis [12-14]. Inflammation in atherosclerosis is modulated by cytokines that differentially affect endothelial dysfunction. Distinct cytokines promote pro- as well as antiatherogenic processes, thus modulating plaque development and clinical outcome $[14,25,26]$. IFN- $\gamma$ and TNF$\alpha$ mediate proatherogenic processes by promoting monocyte activation and by influencing collagen synthesis and expression of adhesion molecules, tissue factor, and matrix metalloproteinases [27, 28].

Our finding on actin-specific T-cell activation is in line with a previous study where we identified actin as a candidate autoantigen of humoral immune response in patients with carotid atherosclerosis [22]. Actin is a globular protein quite abundant in eukaryotic cells. It can polymerize in the presence of ATP and its structure is remarkably conserved during evolution. Anti-actin antibodies have been associated with various autoimmune diseases including systemic lupus erythematosus [29], a disease in which endothelial damage plays a key role. Anti-filamentous actin antibodies characterize autoimmune hepatitis type 1 where the binding domain of $\alpha$ actinin on actin was shown to be a predominant actin epitope [30]. Anti-actin antibodies were also found in $52-85 \%$ of patients with autoimmune hepatitis or chronic active hepatitis, in $22 \%$ of patients with primary biliary cirrhosis, and in 

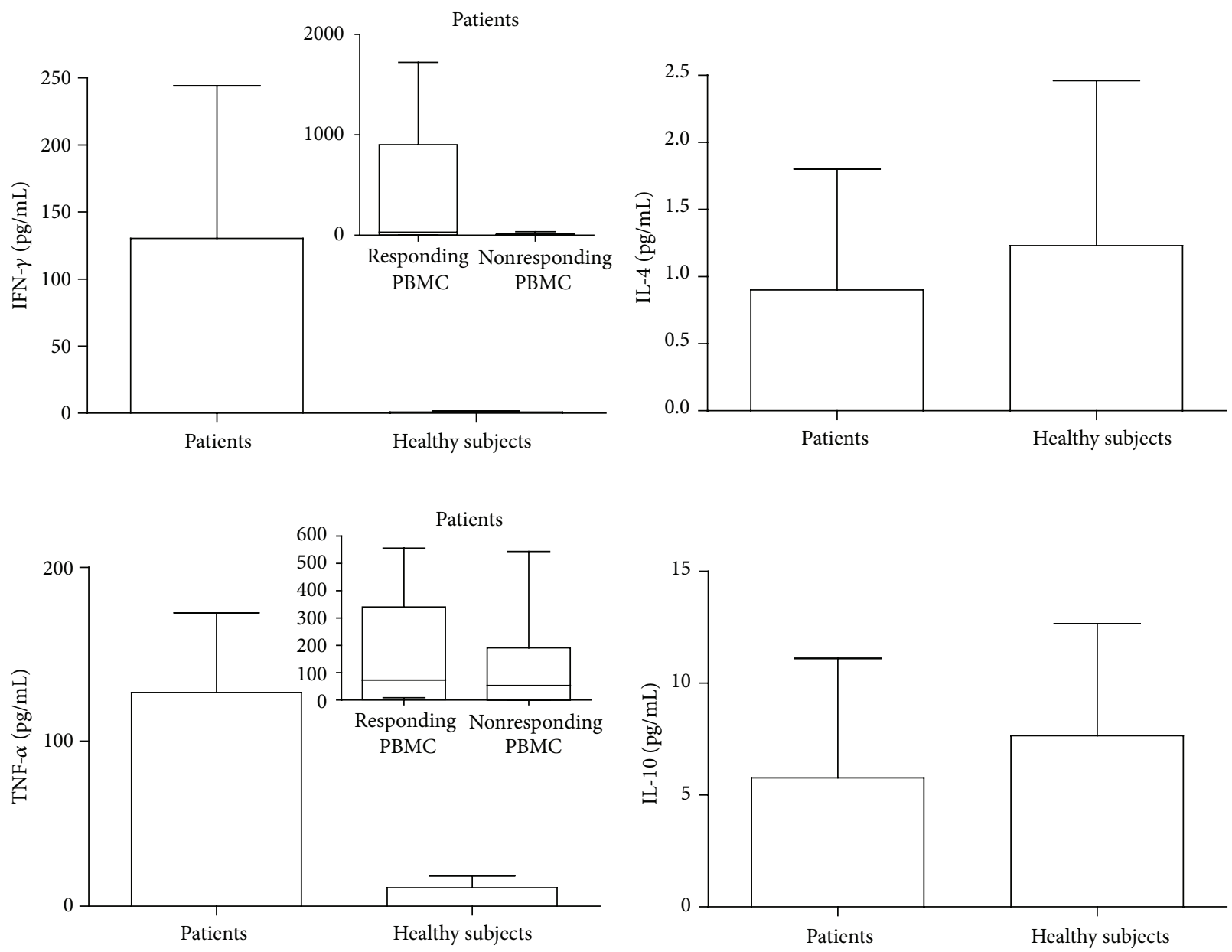

FIGURE 3: Cytokine secretion in culture supernatants from patients' and healthy subjects' peripheral blood mononuclear cell (PBMC) samples; IFN- $\gamma$ and TNF- $\alpha$ production by patients' PBMC responding or not to actin in proliferation assay.

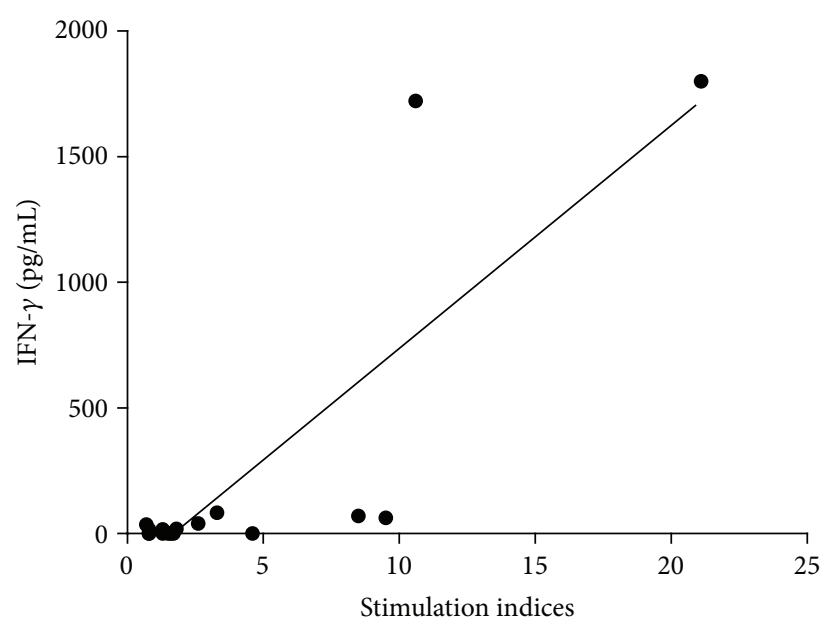

FIGURE 4: Positive correlation between IFN- $\gamma$ concentrations and proliferative response to actin (stimulation indices) in patients with carotid atherosclerosis. $P<10^{-4} ; r=0.71$. 
patients with celiac disease and with autoimmune haemolytic anaemia [31-34]. Furthermore, nonmuscle $\alpha$-actinin 4 and cytoplasmic $\beta$-actin were identified as immunodominant ovarian autoantigens involved in ovarian autoimmunity [35].

An interesting and extremely important aspect in autoimmune diseases is to understand how abundant and highly conserved self-proteins can become the antigenic target of autoimmune reactions. One of the mechanisms breaking tolerance to self could be apoptosis. It has been shown that apoptotic cancer cells may render actin immunogenic by exposing it on their surfaces [36, 37]. In addition, many autoantigens, and in particular actin, represent a substrate for the proapoptotic cysteine proteases. The polypeptides produced in this way can be released into the extracellular space or can be presented as neoantigens, thus generating an autoimmune response [38, 39]. Interestingly, several studies have shown the presence of apoptotic cells, particularly macrophages and smooth muscle cells, in all stages of atherosclerosis development $[40,41]$.

In our study, we observed a positive association between the presence of diabetes and the response to actin. Pancreatic beta-cell death by apoptosis, which can be induced by multiple stresses, contributes significantly to the pathogenesis of type 2 diabetes [42]. The possibility that diabetes, characterized by increasing oxidative stress and apoptosis, may trigger the autoimmune response to actin is interesting and needs further investigation.

A limitation of our study is that it does not provide a causal association between the T-cell response to actin and atherosclerosis in humans. In vivo experimental models are required to address this question.

\section{Conclusions}

Our study takes research into the involvement of autoimmune responses in the pathogenesis of atherosclerosis, a small step ahead indicating actin as a candidate autoantigen target of cell-mediated immune responses in a proportion of patients with carotid atherosclerosis. Our findings here call for further studies to identify epitopes on actin recognized by specific T lymphocytes. The identification of these epitopes might be useful to design novel preventive strategies.

\section{References}

[1] A. C. Van der Wal, J. J. Piek, O. J. de Boer et al., "Recent activation of the plaque immune response in coronary lesions underlying acute coronary syndromes," Heart, vol. 80, no. 1, pp. $14-18,1998$.

[2] J. Frostegård, A.-K. Ulfgren, P. Nyberg et al., "Cytokine expression in advanced human atherosclerotic plaques: dominance of pro-inflammatory (Th1) and macrophage-stimulating cytokines," Atherosclerosis, vol. 145, no. 1, pp. 33-43, 1999.

[3] O. J. De Boer, A. C. van der Wal, M. A. Houtkamp, J. M. Ossewaarde, P. Teeling, and A. E. Becker, "Unstable atherosclerotic plaques contain T-cells that respond to Chlamydia pneumoniae," Cardiovascular Research, vol. 48, no. 3, pp. 402-408, 2000.
[4] Y. Shoenfeld, R. Gerli, A. Doria et al., "Accelerated atherosclerosis in autoimmune rheumatic diseases," Circulation, vol. 112, no. 21, pp. 3337-3347, 2005.

[5] N. Bassi, A. Ghirardello, L. Iaccarino et al., "OxLDL/ $\beta 2$ GPIanti-oxLDL/ $\beta 2$ GPI complex and atherosclerosis in SLE patients," Autoimmunity Reviews, vol. 7, no. 1, pp. 52-58, 2007.

[6] H. Zinger, Y. Sherer, and Y. Shoenfeld, "Atherosclerosis in autoimmune rheumatic diseases-mechanisms and clinical findings," Clinical Reviews in Allergy and Immunology, vol. 37, no. 1, pp. 20-28, 2009.

[7] E. Matsuura, K. Kobayashi, and L. R. Lopez, "Preventing autoimmune and infection triggered atherosclerosis for an enduring healthful lifestyle," Autoimmunity Reviews, vol. 7, no. 3, pp. 214-222, 2008.

[8] J. Nilsson and G. K. Hansson, "Autoimmunity in atherosclerosis: a protective response losing control?" Journal of Internal Medicine, vol. 263, no. 5, pp. 464-478, 2008.

[9] N. R. Madamanchi, A. Vendrov, and M. S. Runge, "Oxidative stress and vascular disease," Arteriosclerosis, Thrombosis, and Vascular Biology, vol. 25, no. 1, pp. 29-38, 2005.

[10] T. W. Stief, J. Kropf, V. Kretschmer, M. O. Doss, and J. Fareed, "Singlet oxygen (1O2) Inactivates plasmatic free and complexed a2-macroglobulin," Thrombosis Research, vol. 98, no. 6, pp. 541$547,2000$.

[11] S. Stemme, B. Faber, J. Holm, O. Wiklund, J. L. Witztum, and G. K. Hansson, "T lymphocytes from human atherosclerotic plaques recognize oxidized low density lipoprotein," Proceedings of the National Academy of Sciences of the United States of America, vol. 92, no. 9, pp. 3893-3897, 1995.

[12] B. Buttari, E. Profumo, V. Mattei et al., "Oxidized $\beta 2$-glycoprotein I induces human dendritic cell maturation and promotes a T helper type 1 response," Blood, vol. 106, no. 12, pp. 3880-3887, 2005.

[13] B. Buttari, E. Profumo, L. Petrone et al., "Free hemoglobin: a dangerous signal for the immune system in patients with carotid atherosclerosis?" Annals of the New York Academy of Sciences, vol. 1107, pp. 42-50, 2007.

[14] E. Profumo, B. Buttari, and R. Riganò, "Oxidized haemoglobin as antigenic target of cell-mediated immune reactions in patients with carotid atherosclerosis," Autoimmunity Reviews, vol. 8, no. 7, pp. 558-562, 2009.

[15] M. Benagiano, M. M. D’Elios, A. Amedei et al., "Human 60-kDa heat shock protein is a target autoantigen of T cells derived from atherosclerotic plaques," Journal of Immunology, vol. 174, no. 10, pp. 6509-6517, 2005.

[16] Y. Döring, H. D. Manthey, M. Drechsler et al., "Auto-antigenic protein-DNA complexes stimulate plasmacytoid dendritic cells to promote atherosclerosis," Circulation, vol. 125, no. 13, pp. 1673-1683, 2012.

[17] W. Martinet, D. M. Schrijvers, and G. R. Y. De Meyer, "Necrotic cell death in atherosclerosis," Basic Research in Cardiology, vol. 106, no. 5, pp. 749-760, 2011.

[18] I. Tabas, "Macrophage apoptosis in atherosclerosis: consequences on plaque progression and the role of endoplasmic reticulum stress," Antioxidants and Redox Signaling, vol. 11, no. 9, pp. 2333-2339, 2009.

[19] C. D. Gregory and A. Devitt, "The macrophage and the apoptotic cell: an innate immune interaction viewed simplistically?" Immunology, vol. 113, no. 1, pp. 1-14, 2004.

[20] A. Hochreiter-Hufford and K. S. Ravichandran, "Clearing the dead: apoptotic cell sensing, recognition, engulfment, and 
digestion," Cold Spring Harbor Perspectives in Biology, vol. 5, no. 1, Article ID a008748, 2013.

[21] L.-P. Erwig and P. M. Henson, "Immunological consequences of apoptotic cell phagocytosis," American Journal of Pathology, vol. 171, no. 1, pp. 2-8, 2007.

[22] P. Margutti, F. Delunardo, M. Sorice et al., "Screening of a HUAEC cDNA library identifies actin as a candidate autoantigen associated with carotid atherosclerosis," Clinical and Experimental Immunology, vol. 137, no. 1, pp. 209-215, 2004.

[23] H. C. Stary, A. B. Chandler, R. E. Dinsmore et al., "A definition of advanced types of atherosclerotic lesions and a histological classification of atherosclerosis: a report from the Committee on Vascular Lesions of the Council on Arteriosclerosis, American Heart Association," Arteriosclerosis, Thrombosis, and Vascular Biology, vol. 15, no. 9, pp. 1512-1531, 1995.

[24] R. Riganò, E. Profumo, F. Bruschi et al., "Modulation of human immune response by Echinococcus granulosus antigen $B$ and its possible role in evading host defenses," Infection and Immunity, vol. 69, no. 1, pp. 288-296, 2001.

[25] M. Benagiano, A. Azzurri, A. Ciervo et al., "T helper type 1 lymphocytes drive inflammation in human atherosclerotic lesions," Proceedings of the National Academy of Sciences of the United States of America, vol. 100, no. 11, pp. 6658-6663, 2003.

[26] E. Profumo, A. Siracusano, E. Ortona et al., "Cytokine expression in circulating $\mathrm{T}$ lymphocytes from patients undergoing carotid endarterectomy," Journal of Cardiovascular Surgery, vol. 44, no. 2, pp. 237-242, 2003.

[27] P. E. Szmitko, C.-H. Wang, R. D. Weisel, J. R. de Almeida, T. J. Anderson, and S. Verma, "New markers of inflammation and endothelial cell activation: part I," Circulation, vol. 108, no. 16, pp. 1917-1923, 2003.

[28] H. Ait-Oufella, S. Taleb, Z. Mallat, and A. Tedgui, "Recent advances on the role of cytokines in atherosclerosis," Arteriosclerosis, Thrombosis, and Vascular Biology, vol. 31, no. 5, pp. 969979, 2011.

[29] A. Boulila, J. Hachicha, F. Z. Adyel et al., "Deposition of anti-actin antibodies in the kidney of a patient with systemic lupus erythematosus under immunosuppressive treatment," Nephrology Dialysis Transplantation, vol. 11, no. 12, pp. 24782481, 1996.

[30] G. Paul, D. Georgios, N. Jean-Baptiste et al., "Double reactivity against actin and $\alpha$-actinin defines a severe form of autoimmune hepatitis type 1," Journal of Clinical Immunology, vol. 26, no. 6, pp. 495-505, 2006.

[31] L. Leibovitch, J. George, Y. Levi, R. Bakimer, and Y. Shoenfeld, "Anti-actin antibodies in sera from patients with autoimmune liver diseases and patients with carcinomas by ELISA," Immunology Letters, vol. 48, no. 2, pp. 129-132, 1995.

[32] A. N. Hamlyn and P. A. Berg, "Haemagglutinating anti-actin antibodies in acute and chronic liver disease," Gut, vol. 21, no. 4, pp. 311-317, 1980.

[33] G. Samaşca, A. Băican, T. Pop et al., "IgG-F-actin antibodies in celiac disease and dermatitis herpetiformis," Roumanian Archives of Microbiology and Immunology, vol. 69, no. 4, pp. 177182, 2010.

[34] K. M. Felder, K. Hoelzle, K. Heinritzi, M. Ritzmann, and L. E. Hoelzle, "Antibodies to actin in autoimmune haemolytic anaemia," BMC Veterinary Research, vol. 6, article 18, 2010.

[35] P. V. Mande, F. R. Parikh, I. Hinduja et al., "Identification and validation of candidate biomarkers involved in human ovarian autoimmunity," Reproductive BioMedicine Online, vol. 23, no. 4, pp. 471-483, 2011.
[36] M. H. Hansen, H. Nielsen, and H. J. Ditzel, "The tumorinfiltrating B cell response in medullary breast cancer is oligoclonal and directed against the autoantigen actin exposed on the surface of apoptotic cancer cells," Proceedings of the National Academy of Sciences of the United States of America, vol. 98, no. 22, pp. 12659-12664, 2001.

[37] M. H. Hansen, H. V. Nielsen, and H. J. Ditzel, “Translocation of an intracellular antigen to the surface of medullary breast cancer cells early in apoptosis allows for an antigen-driven antibody response elicited by tumor-infiltrating B cells," Journal of Immunology, vol. 169, no. 5, pp. 2701-2711, 2002.

[38] C. Kayalar, T. Örd, M. P. Testa, L.-T. Zhong, and D. E. Bredesen, "Cleavage of actin by interleukin $1 \beta$-converting enzyme to reverse DNase I inhibition," Proceedings of the National Academy of Sciences of the United States of America, vol. 93, no. 5, pp. 2234-2238, 1996.

[39] M. Piacentini and V. Colizzi, "Tissue transglutaminase: apoptosis versus autoimmunity," Immunology Today, vol. 20, no. 3, pp. 130-134, 1999.

[40] Z. Mallat and A. Tedgui, "Apoptosis in the vasculature: mechanisms and functional importance," British Journal of Pharmacology, vol. 130, no. 5, pp. 947-962, 2000.

[41] M. M. Kockx and A. G. Herman, "Apoptosis in atherosclerosis: beneficial or detrimental?" Cardiovascular Research, vol. 45, no. 3, pp. 736-746, 2000.

[42] J. D. Johnson and D. S. Luciani, "Mechanisms of pancreatic $\beta$-cell apoptosis in diabetes and its therapies," Advances in Experimental Medicine and Biology, vol. 654, pp. 447-462, 2010. 


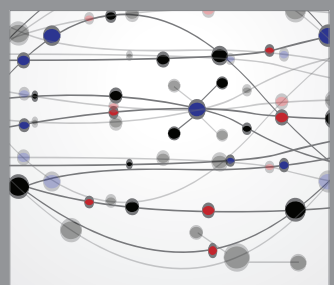

The Scientific World Journal
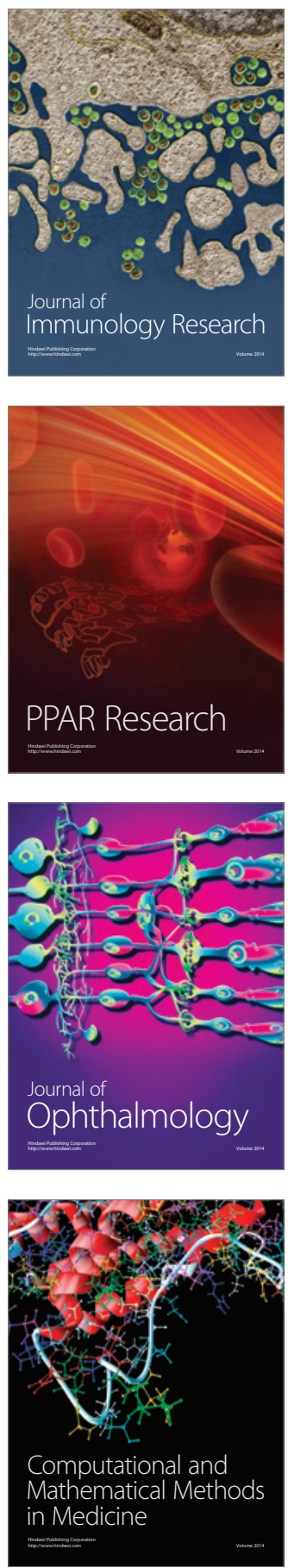

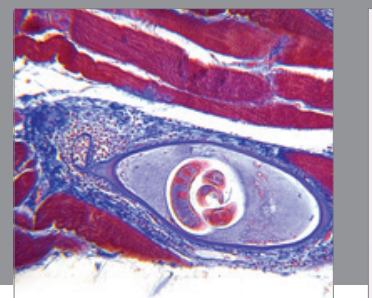

Gastroenterology

Research and Practice
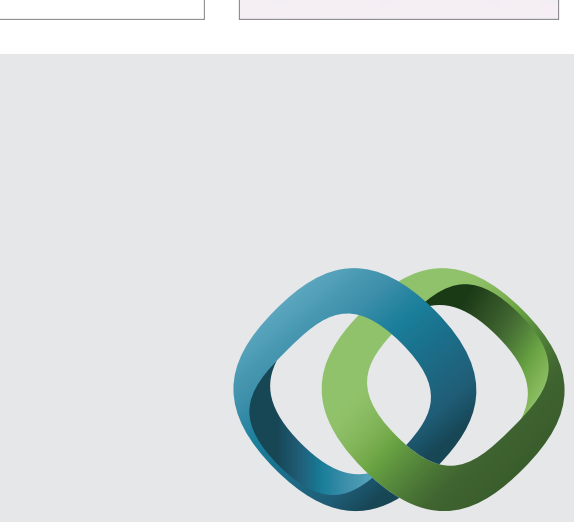

\section{Hindawi}

Submit your manuscripts at

http://www.hindawi.com
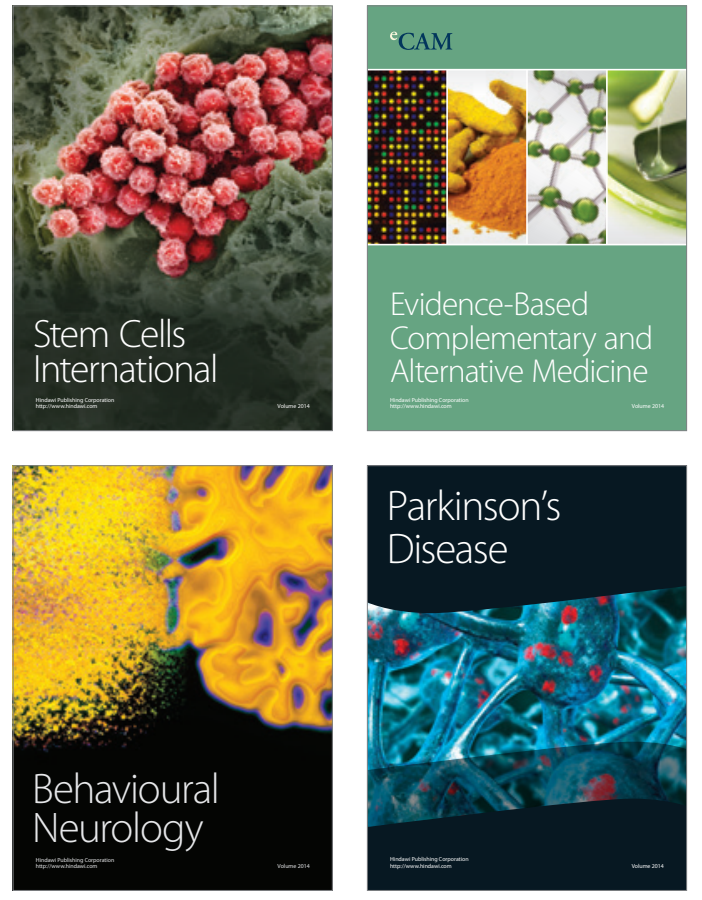
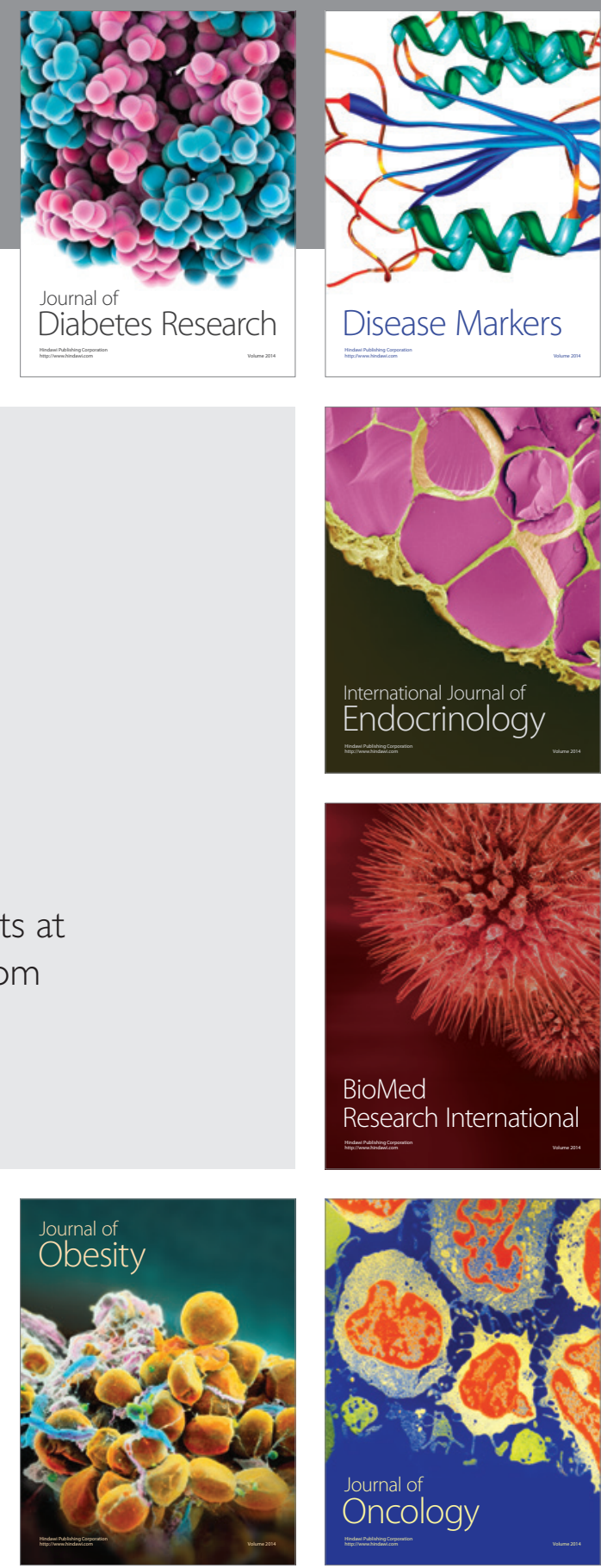

Disease Markers
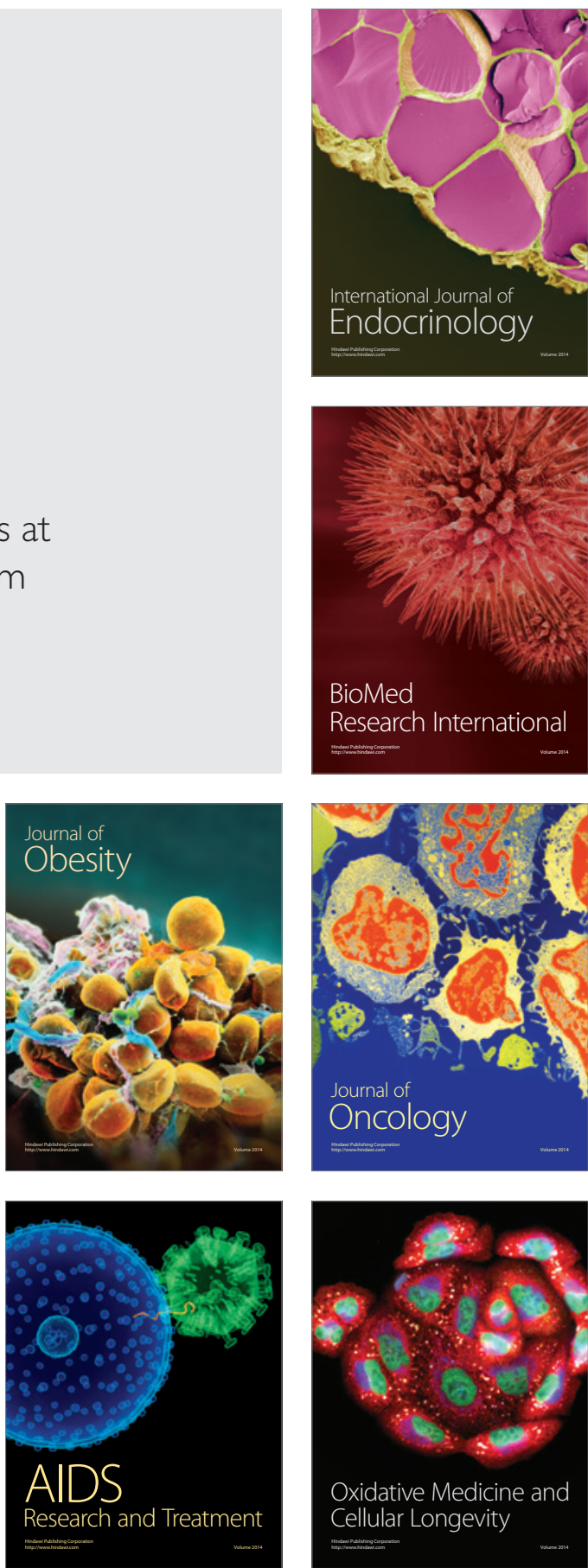